les "montagnes" ne pratiquant plus la traite à cette époque : 90 p. 100 du total environ $(\mathrm{N}=$ 2 132), en fonction de la qualité de la "montagne " appréciée subjectivement d'après le milieu naturel ou artificiel d'élevage (apport d'aliments concentrés) : 4 classes ont été considérées. Les différences de croissance journalière moyenne (Aubrac $\times$ charolais vs Aubrac $\times$ Aubrac) étaient respectivement dans l'ordre de qualité décroissante des montagnes de $+178 \mathrm{~g},+107 \mathrm{~g},+106 \mathrm{~g}$ et $+24 \mathrm{~g}$ pour les $\widehat{\delta} \delta ;+121 \mathrm{~g},+60 \mathrm{~g},+54 \mathrm{~g}$ et $-38 \mathrm{~g}$ pour les 우우. Ces résultats indiquent encore une interaction croisement $\times$ milieu d'élevage dans les montagnes où le veau consomme la totalité du lait de sa mère. Cette même année, on a pu en outre comparer sur des montagnes de bonne qualité la croissance de veaux Aubrac $\times$ Aubrac, Aubrac $\times$ charolais, croisés de retour sur charolais et charolais $\times$ charolais soit des génotypes possédant 0,50, 75 et 100 p. 100 de sang charolais. Les valeurs moyennes de croissance journalière étaient respectivement pour ces 4 catégories de $995 \mathrm{~g}, 1013 \mathrm{~g}, 1009 \mathrm{~g}$, et $902 \mathrm{~g}$ chez les ડ̋ð; de $778 \mathrm{~g}, 767 \mathrm{~g}, 806 \mathrm{~g}, 746 \mathrm{~g}$ chez les 우우.

Tous ces résultats traduisent des différences génétiques d'adaptation au milieu (maternel pour le veau, physique pour le couple mère-veau) qu'il convient d'analyser. Ils conduisent à comparer l'efficacité économique de diverses stratégies d'exploitation des croisements entre le cheptel autochtone et les mâles de races à viande, à spécialiser enfin l'élevage dans chaque zone en fonction du génotype le mieux adapté aux caractéristiques de la zone. On a pu observer d'ores et déjà sur le terrain des directions privilégiées d'extension du croisement, directions qui sont fonction des potentialités du milieu.

Sous notre direction, cette recherche a fait l'objet d'un document de fin d'éludes de l'École supérieure d'Agriculture de Purpan par A. Gauthier (1967, ronéoté). Elle s'intègre par ailleurs dans le cadre des travaux de la R.C.P. Aubrac (Recherche coopérative sur Programme) organisés par le C.N.R.S.

\title{
UTILISATION DE TAUREAUX CHAROLAIS, LIMOUSINS ET PIÉMONTAIS POUR LE CROISEMENT INDUSTRIEI EN SARDAIGNE
}

\author{
S. Casu. - Istituto Zootecnico e Caseario per la Sardegna, Sassari.
}

Durant 3 années (1964-65-66), 150 vaches de races sardo-modicana, bruno-sarda et métisse ont été accouplées à 4 taureaux charolais, 2 limousins et 4 piémontais pour la production de veaux de 15 mois ("vitellone "). Après un sevrage à 6 mois, les produits croisés contrôlés (85 charolais, 89 limousins et 74 piémontais) ont reçu une ration à base de foin et d'aliment concentré jusqu'à l'abattage. Par une analyse de moindres carrés, on a montré la supériorité pondérale des mâles à tout âge par rapport aux femelles : 1,600 kg à la naissance, 8,270 kg au sevrage, $41,100 \mathrm{~kg}$ à 12 mois, $81,700 \mathrm{~kg}$ à l'abattage, $52,800 \mathrm{~kg}$ en carcasse (avec $3,80 \%$ et $0,54 \%$ de gras et d'os en moins). L'année de naissance a une influence significative sur le poids à tous les âges et sur le poids et la qualité des carcasses. Les produits des vaches bruno-sarda et sardo-modicana ont. une meilleure vitesse de croissance et des carcasses plus lourdes que les produits des vaches métisses. La race des taureaux n'a pas d'influence sur le poids au sevrage, mais à l'abattage les veaux croisés charolais sont plus lourds de 20 kilogrammes que les autres. La différence des poids de carcasses n'est toutefois que de 10 kilogrammes : les croisés piémontais ont le meilleur rendement à l'abattage, leur carcasse étant de meilleure qualité (gras et os moins importants, pourcentage plus élevé de morceaux nobles), supériorité due en partie au caractère culard des taureaux piémontais utilisés. Les taureaux charolais et piémontais en croisement industriel avec des vaches locales donnent donc des carcasses de meilleure valeur bouchère (poids et qualité) que les taureaux limousins et se révèlent, par conséquent, les plus intéressants pour la production de "vitellone " dans les conditions moyennes d'élevage de la Sardaigne. 